\title{
POROUS MATERIAL EFFECT ON GEARBOX VIBRATION AND ACOUSTIC BEHAVIOR
}

\author{
Mohamed Riadh Letaief, Lassaad Walha, Mohamed Taktak, \\ Fakher ChaAri, Mohamed Haddar \\ Mechanical, Modeling and Manufacturing Laboratory LA2MP, National School of Engineers of Sfax, Sfax, Tunisia \\ e-mail: walhalassaad@yahoo.fr
}

\begin{abstract}
In this paper, we define a resolution method to study the effect of a porous material on vibro-acoustic behavior of a geared transmission. A porous plate is coupled with the gearbox housing cover. The developed model depends on the gearbox characteristic and poroelastic parameters of the porous material. To study the acoustic effect of the housing cover, the acoustic transmission loss is computed by simulating numerically the elastic-porous coupled plate model, and the numerical implementation is performed by directly programming the mixed displacement-pressure formulation. To study the vibration effect, the bearing displacement is computed using a two-stage gear system dynamical model and used as the gearbox cover excitation. Numerical implementation is performed by direct programming of the Leclaire formulation.
\end{abstract}

Keywords: porous material, gearbox, vibro-acoustic behavior

\section{Introduction}

Controlling the vibro-acoustic behavior of rotating machinery has become a quality factor to improve the comfort by reducing noise and vibration levels. One of the major noise and vibration sources are geared transmissions (gears, shafts, roller bearings and the housing). The generalized forces which generate the vibration response of the gearbox housing are multiple, as expressed by Remond et al. (1993). Sources of vibration excitations generated by geared transmissions can be divided into two categories, first the internal excitation sources like the static transmission error under load, elastic deformations of teeth, fluctuation in the frictional force developed by Houser (1991), Aziz and Seirg (1994), schock phenomenon and the projection or flows of the lubricant on walls of the housing according to Houser (1991) and Houjoh and Umezawa (1992). External sources of excitation can be associated with the fluctuations in engine torque and load inertia.

Regardless of directivity of the source, larger walls of the housing are more flexible and contribute most to noise radiation. A parametric study performed by Sibe (1997) shows that the more walls are heavy, stiff and thick, the higher is the acoustic transmission loss of the housing. An increase in the thickness of the housing is unfortunately contrary to the desire of manufacturers who always want to increase the specific power of their transmissions. Note that in the majority of gearboxes, their housings covers are more flexible than other parts body of the housing and have the largest surface of acoustic radiation while looking for a method how to decrease their acoustic emission, some research work as that carried out by Guezzen (2004), confirmed effects of structure of the gearbox cover on noise radiation. In this context, we study a housing cover of a gearbox coupled with a porous material plate to isolate sources of noise radiation.

Various models have been developed to describe the acoustic propagation in porous media. One of the best known and the easiest to implement is the model of Delany and Bazley (1970). 
However, this model is limited because it represents only tested materials and does not express the phenomenon related to skeleton vibrations. To model more accurately the dissipative effects, unlike in the model developed by Johnson et al. (1987), one may introduce a function of viscous form which is not limited by the geometric nature of the skeleton. Modeling of the variation of the viscous dissipation modulus may require introduction of the viscous characteristic length which is an intrinsic parameter of the material that can be obtained through experience. Similarly, Champoux and Allard (1991) defined the thermal characteristic length as an intrinsic parameter expressing thermal effects. Lafarge et al. (1997) introduced thermal permeability to improve thermal effects at low frequencies. However, the model with a rigid structure is not suitable when the skeleton of the material is deformed or mobile: this is the case in many applications where a porous material is directly subjected to a mechanical or acoustic wave excitation which is the subject of our paper. Allard (1993) adapted a model for acoustic applications by integrating various contributions previously cited, see Johnson et al. (1987), Champoux and Allard (1991) and Lafarge et al. (1997). This model, commonly called the Biot-Allard model is used in our study since porous materials are subjected to the imposed displacement or acoustic pressure.

In Section 2, we describe equations of motion for the dynamic model of gearbox and the housing cover (elastic and porous coupled plate) implementing porous models. In Section 3, we present the resolution method (input and output, geometry, implemented porous and boundary conditions). In Section 4, we describe the porous plate effect on vibration and the acoustic transmission loss of our gearbox housing cover by a study case.

\section{Gearbox modelling}

In most gearboxes, especially those having reduced sizes, the wheel axis is in the same plane between the two parts of the gearbox (Fig. 1) that enables easy assembling of the wheels.

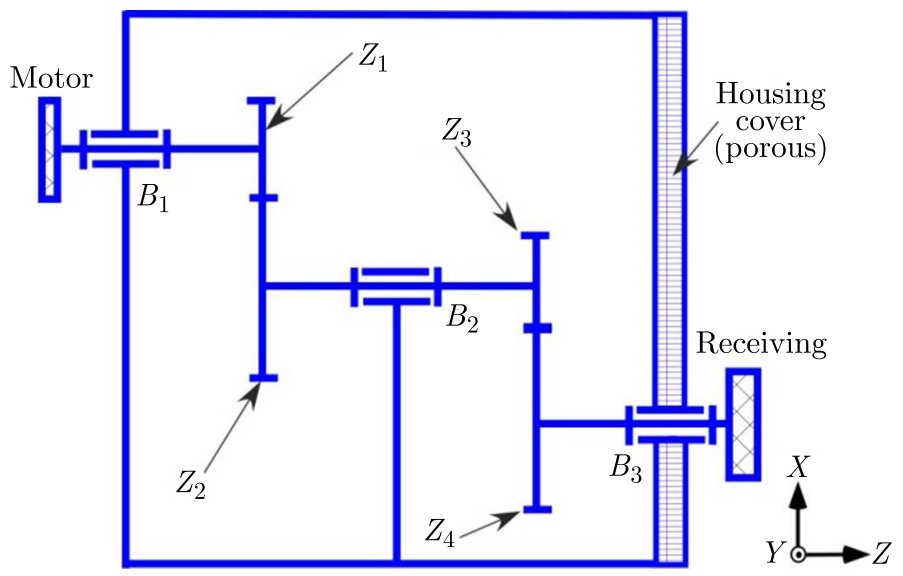

Fig. 1. Plane configuration of a two-stage gear system and the porous housing cover

We defined a fixed reference frame $\left(O, X_{0}, Y_{0}\right)$ in the model. $\alpha_{i}$ are pressure angles of two gearmesh contact. In this paper, these angles are equal to $20^{\circ}$ in the case of the gearings with right teeth.

\subsection{Dynamic model of a two-stage gear system}

A two-stage gear system is composed of two trains of gearings. Every train links two blocks. So, the gear system has in total three blocks $(j=1,2,3)$. Every block is supported by a flexible bearing whose bending stiffness is $k_{x j}$ and the traction compression stiffness is $k_{y j}$. The dynamic 
model developed has twelve degrees of freedom: six angular movements $\gamma_{j i}$ and six linear movements $x_{j}$ and $y_{j}$ (Fig. 2). The motor and receiving wheels are introduced by inertias $I_{m}$ and $I_{r}$ as expressed by Miller (1999) with the assumption that we use short shafts. The other spur gears constitute the gearbox. The gearmeshes are modeled by a linear spring $k s(t)(s=1,2)$ along the lines of action represented in Fig. 2. $\alpha_{i}$ are pressure angles of two gearmesh contact. The angular displacements of every wheel are noticed by $\gamma_{j i}$ with the indices $j=1$ to 3 designating the number of the block, and $i=1,2$ designating the two wheels of each block. Besides, the linear displacements of the bearing denoted by $x_{j}$ and $y_{j}$ are measured in the plane which is orthogonal to the axis of wheel rotation.

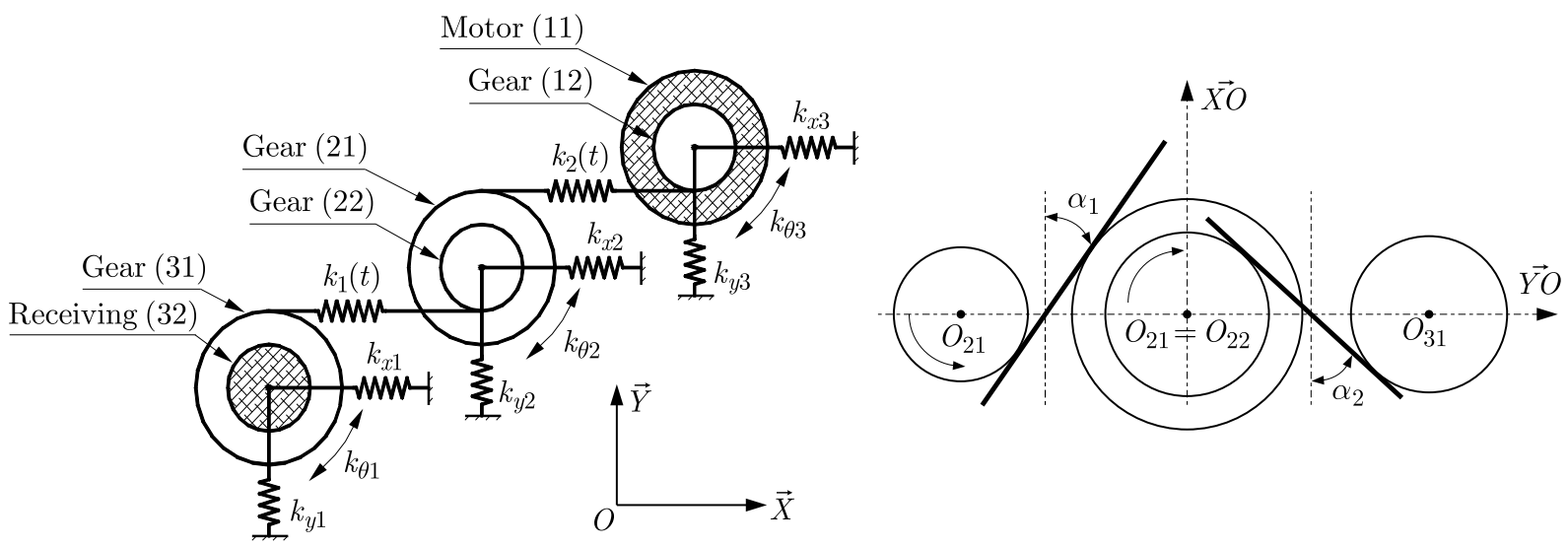

Fig. 2. Model of the two-stage gear system developed by Walha et al. (2009)

\subsection{Modeling of the mesh stiffness}

Generally, we can model variation of the gearmesh stiffness $k_{i}(t)$ by a square wave which was developed by Velex (1988). The variation in stiffness comes from the fact that during meshing there is a change in the number of contacting pairs. For spur gears, there is a change for two pairs of teeth in contact for a period of meshing. The square wave variation is the best representative of the real phenomenon, and is represented in Fig. 3.

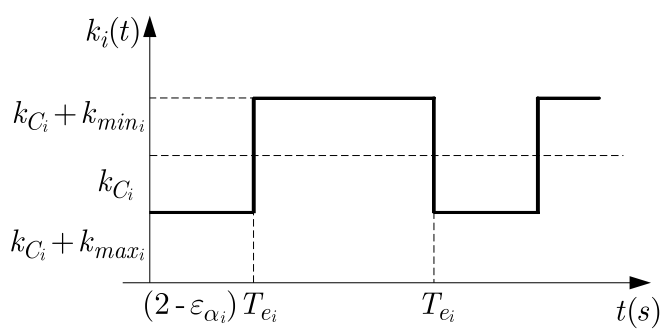

Fig. 3. Modeling of the mesh stiffness variation

The gearmesh stiffness variation can be decomposed into two components: an average component denoted by $k_{c_{i}}$, and a time-dependent one denoted by $k_{v_{i}}(t)$.

The extreme values of the mesh stiffness are defined by

$$
k_{\min _{i}}=-\frac{k_{c}}{2 \varepsilon_{\alpha i}} \quad k_{\max _{i}}=-k_{\min _{i}} \frac{2-\varepsilon_{\alpha i}}{\varepsilon_{\alpha i}-1}
$$

The terms $\varepsilon_{\alpha i}$ are the contact ratio corresponding to the two gearmesh contacts. 


\subsection{Equations of motion}

Applying the Lagrange equations, we obtain a system of differential equations governing the dynamic behavior. It can be written in the following usual matrix form

$$
\mathbf{M} \ddot{\mathbf{q}}+\left[\mathbf{K}_{s}+\mathbf{K}(t)\right] \mathbf{q}=\mathbf{F}_{0}
$$

where $\mathbf{q}$ is the generalized coordinate vector, $\mathbf{M}$ is the mass matrix expressed by

$$
\mathbf{M}=\operatorname{diag}\left(m_{1}, m_{1}, m_{2}, m_{2}, m_{3}, m_{3}, I_{m}, I_{12}, I_{21}, I_{22}, I_{31}, I_{r}\right)
$$

$m_{j}$ is mass of the block $j, I_{m}$ is the polar inertia of the motor wheel, $I_{r}$ is the polar inertia of the receiving wheel.

The matrix of average stiffness of the structure is defined by

$$
\begin{aligned}
\mathbf{K}_{s}= & {\left[\begin{array}{cc}
\mathbf{K}_{p} & \mathbf{0} \\
\mathbf{0} & \mathbf{K}_{\theta}
\end{array}\right] } \\
\mathbf{K}_{p}= & {\left[\begin{array}{cccccc}
k_{x 1} & 0 & 0 & 0 & 0 & 0 \\
0 & k_{y 1} & 0 & 0 & 0 & 0 \\
0 & 0 & k_{x 2} & 0 & 0 & 0 \\
0 & 0 & 0 & k_{y 2} & 0 & 0 \\
0 & 0 & 0 & 0 & k_{x 3} & 0 \\
0 & 0 & 0 & 0 & 0 & k_{y 3}
\end{array}\right] } \\
\mathbf{K}_{\theta}= & {\left[\begin{array}{cccccc}
k_{\theta 1} & -k_{\theta 1} & 0 & 0 & 0 & 0 \\
-k_{\theta 1} & k_{\theta 1} & 0 & 0 & 0 & 0 \\
0 & 0 & k_{\theta 2} & -k_{\theta 2} & 0 & 0 \\
0 & 0 & -k_{\theta 2} & k_{\theta 2} & 0 & 0 \\
0 & 0 & 0 & 0 & k_{\theta 3} & -k_{\theta 3} \\
0 & 0 & 0 & 0 & -k_{\theta 3} & k_{\theta 3}
\end{array}\right] }
\end{aligned}
$$

where $\mathbf{K}_{p}$ is the bearing stiffness matrix and $\mathbf{K}_{\theta}$ is the shaft torsional stiffness matrix.

$\mathbf{K}(t)$ is the stiffness matrix of the engagement which is variable over time

$$
\mathbf{K}(t)=\left[\begin{array}{cc}
\mathbf{K}_{1}(t) & \mathbf{K}_{12}(t) \\
\mathbf{K}_{12}^{\mathrm{T}}(t) & \mathbf{K}_{2}(t)
\end{array}\right]
$$

where

$$
\begin{aligned}
\mathbf{K}_{1}(t)= & {\left[\begin{array}{cccccc}
k_{1} s_{1}^{2} & -k_{1} s c_{1} & -k_{1} s_{1}^{2} & k_{1} s c_{1} & 0 & 0 \\
-k_{1} s c_{1} & k_{1} c_{1}^{2} & k_{1} s c_{1} & -k_{1} c_{1}^{2} & 0 & 0 \\
-k_{1} s_{1}^{2} & k_{1} s c_{1} & k_{1} s_{1}^{2}+k_{2} s_{2}^{2} & -k_{1} s c_{1}-k_{2} s c_{2} & -k_{2} s_{2}^{2} & k_{2} s c_{2} \\
k_{1} s c_{1} & -k_{1} c_{1}^{2} & -k_{1} s c_{1}-k_{2} s c_{2} & k_{1} c_{1}^{2}+k_{2} c_{2}^{2} & k_{2} s c_{2} & -k_{2} c_{2}^{2} \\
0 & 0 & -k_{2} s_{2}^{2} & k_{2} s c_{2} & k_{2} s_{2}^{2} & -k_{2} s c_{2} \\
0 & 0 & k_{2} s c_{2} & -k_{2} c_{2}^{2} & -k_{2} s c_{2} & k_{2} c_{2}^{2}
\end{array}\right] } \\
\mathbf{K}_{12}(t) & =\left[\begin{array}{cccccc}
0 & -k_{1} r_{b 12} s_{1} & -k_{1} r_{b 21} s_{1} & k_{1} s c_{1} & 0 & 0 \\
0 & k_{1} r_{b 12} c_{1} & k_{1} r_{b 21} c_{1} & -k_{1} c_{1}^{2} & 0 & 0 \\
0 & k_{1} r_{b 12} s_{1} & k_{1} r_{b 12} s_{1} & -k_{1} s c_{1}-k_{2} s c_{2} & -k_{2} s_{2}^{2} & k_{2} s c_{2} \\
0 & -k_{1} r_{b 12} c_{1} & -k_{1} s c_{1}-k_{2} s c_{2} & k_{1} c_{1}^{2}+k_{2} c_{2}^{2} & k_{2} s c_{2} & -k_{2} c_{2}^{2} \\
0 & 0 & -k_{2} s_{2}^{2} & k_{2} s c_{2} & k_{2} s_{2}^{2} & -k_{2} s c_{2} \\
0 & 0 & k_{2} s c_{2} & -k_{2} c_{2}^{2} & -k_{2} s c_{2} & k_{2} c_{2}^{2}
\end{array}\right]
\end{aligned}
$$




$$
\mathbf{K}_{2}(t)=\left[\begin{array}{cccccc}
0 & 0 & 0 & 0 & 0 & 0 \\
0 & k_{1} r_{b 12}^{2} & k_{1} r_{b 12} r_{b 21} & 0 & 0 & 0 \\
0 & k_{1} r_{b 12} r_{b 21} & k_{1} r_{b 21}^{2} & 0 & -k_{2} s_{2}^{2} & 0 \\
0 & 0 & 0 & k_{2} r_{b 22}^{2} & k_{2} r_{b 22} r_{b 31} & 0 \\
0 & 0 & 0 & k_{2} r_{b 22} r_{b 31} & k_{2} r_{b 31}^{2} & 0 \\
0 & 0 & 0 & 0 & 0 & 0
\end{array}\right]
$$

where $r_{b}$ is the base radius; $s_{i}, s c_{i}$ and $c_{i}^{2}$ are simplifications of the functions: $s_{i}=\sin ^{2} \phi_{i}$, $s c_{i}=\sin \phi_{i} \cos \phi_{i}$ and $c_{i}^{2}=\cos ^{2} \phi_{i}$, respectively. $\mathbf{F}_{0}$ is the vector of external static forces and can be expressed as

$$
\mathbf{F}_{0}=\left[0,0,0,0,0,0, C_{m}, 0,0,0,0,-C_{r}\right]^{\mathrm{T}}
$$

$C_{m}$ and $C_{r}$ are the motor and receiving wheel torques, respectively.

\section{Modelling of the housing cover}

In our study, the housing cover is modeled as an elastic and porous coupled plate. In fact, two porous models are implemented.

\subsection{Leclaire's formulation}

Leclaire's formulation is based on the classical theory of homogeneous plates and on the Biot stress-strain relations in an isotropic porous medium with a uniform porosity. The vibrations of a rectangular porous plate can be described by two coupled dynamic equations of equilibrium relating the plate deflection $w_{s}$ and the fluid/solid relative displacement $w$.

In the case of a plate with thickness $h$ and subjected to a load $q$, these two equations can be expressed as

$$
\begin{aligned}
& \left(D+\frac{\phi^{2} \widetilde{\lambda}^{f} h^{3}}{12 \phi^{2}}\right) \nabla^{4} w_{s}+h\left(\rho_{1} \ddot{w}_{s}+\rho_{0} \ddot{w}\right)=q \\
& \frac{\tilde{\lambda}^{f} h}{\phi} \nabla^{2} w_{s}-h\left(\rho_{0} \ddot{w}_{s}+m \ddot{w}\right)=0
\end{aligned}
$$

where $D$ is the flexural rigidity, $\rho_{0}$ - density of the fluid, $\rho_{1}$ - density of the frame, $\phi$ - porosity, $\tilde{\lambda}^{f}$ - material expansion coefficient and $m$ is the mass parameter introduced by Biot (1962) given by

$$
m(\omega)=\frac{\tau(\omega)}{\phi} \rho_{0}
$$

where $\omega$ is the pulsation, $\tau(\omega)$ is the dynamic tortuosity expressed as folows

$$
\tau(\omega)=\tau_{\infty}-j \frac{\sigma \phi}{\rho_{0}} F(\omega) \sqrt{1+\frac{4 \eta \alpha_{\infty}^{2} \rho_{0}}{\sigma^{2} \Lambda^{2} \phi^{2}} j \omega} \quad F(\omega)=\sqrt{1-\mathrm{i} \frac{4 \tau_{\infty}^{2} \kappa^{2} \rho_{0} \omega}{\eta \Lambda^{2} \phi^{2}}}
$$

where $F(\omega)$ is the viscosity correction function introduced by Johnson et al. (1987), $\alpha_{\infty}$ is the tortuosity of pores, $\eta$ is the damping coefficient, $\Lambda$ is the characteristic dimension of pores, $\sigma$ is the flow resistivity.

The space derivatives are written with the help of the operators $\nabla^{4}=\nabla^{2}\left(\nabla^{2}\right)$ and $\nabla^{2}=\partial^{2} / p r t x^{2}+\partial^{2} / \partial y^{2}$ of the system of co-ordinates $(x, y)$ while the double dots denote the second time derivative. 
In the first equation of equilibrium (or plate equation) $\left[D+\phi^{2} \widetilde{\lambda}^{f} h^{3} /\left(12 \phi^{2}\right)\right] \nabla^{4} w_{s}$ represents the internal potential force (per unit surface) within the fluid-saturated plate, while the inertia terms $h \rho_{1} \ddot{w}_{s}$ and $h \rho_{0} \ddot{w}$ and the load $q$ are considered as external forces. Similarly, the internal force associated with the fluid-solid relative displacement may be defined, and is given by $\left(\widetilde{\lambda}^{f} h / \phi\right) \nabla^{2} w_{s}$ while the external forces can be taken as $h m \ddot{w}$ and $h \rho_{0} \ddot{w}_{s}$.

We note that the Leclaire formulation is a $2 \mathrm{D}$ one and the unknown variables are $w_{s}$ and $w$. All terms used in this formulation are based on poroelastic material characteristics.

\subsection{The mixed formulation}

In order to reduce the computation time enlarged by complexity of the problem, mixed formulations $(u, p)$ have been implemented. This formulation was developed by Atalla et al. (1998) using the classical equations of Biot where $u$ represents displacement field of the solid phase and $p$ is the pore pressure. Replacing the displacement of the fluid phase by its pressure allows us to reduce degrees of freedom from 6 to 4 per node, valid only for harmonic motion. It is also accurate in the classical formulation $(u, U)$. The modified equations of equilibrium (for small harmonic oscillations) are expressed as follows

$$
\widehat{\sigma}_{i j / j}^{s} S+\omega^{2} \widetilde{\rho} u_{i}+\widetilde{\gamma} p_{/ i}=0 \quad-\omega^{2} \frac{\widetilde{\rho}_{22} \widetilde{\gamma}}{\phi^{2}} u_{i / i}+\omega^{2} \frac{\widetilde{\rho}_{22}}{\widetilde{\lambda}^{f}} p+p_{/ i i}=0
$$

where $\widehat{\sigma}_{i j}^{s}$ is the stress tensor of the material "in vacuo" (does not depend on the fluid phase). It is written by

$$
\widehat{\sigma}_{i j}^{s}=\hat{\tilde{\lambda}}^{s} \varepsilon_{k k}^{s} \delta_{i j}+2 \mu^{s} \varepsilon_{i j}^{s} \quad \varepsilon_{i j}^{s}=\frac{1}{2}\left(u_{i / j}+u_{j / i}\right)
$$

where $\varepsilon_{i j}^{s}$ is the strain tensor of the skeleton, $\mu^{s}$ is the shear modulus of the porous material. The above equations depend on certain factors: $\hat{\tilde{\lambda}}^{s}, \widetilde{\rho}, \widetilde{\gamma}$ and $\widetilde{\lambda}^{f}$. These are based on intrinsic poroelastic characteristics introduced by Horoshenkov and Swift (2001) and Umnova et al. (2001).

\section{Resolution method}

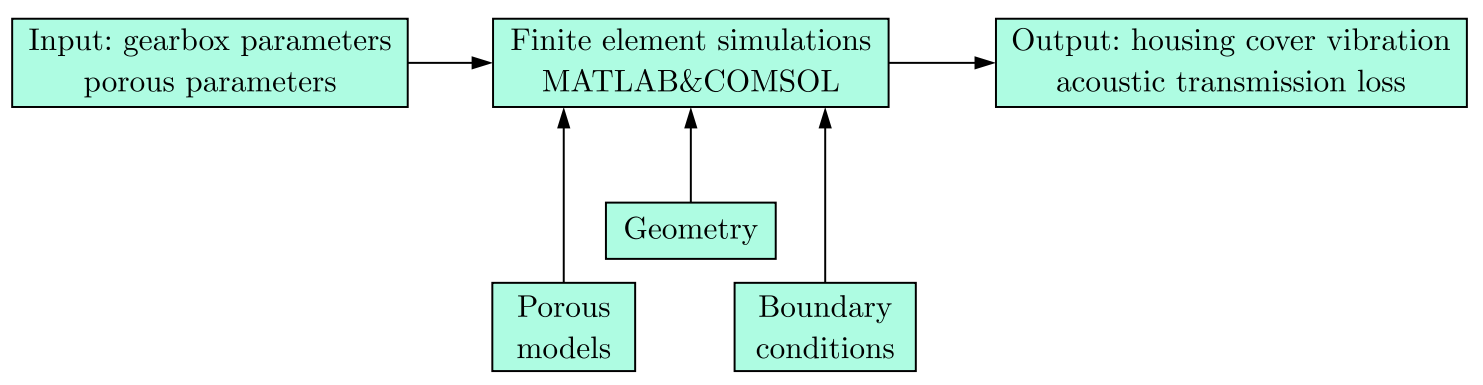

Fig. 4. SADT diagram

For the two cases of study (acoustic and vibration behavior), the implemented porous models are analysed by the finite element software COMSOL and MATLAB. The equations of motion are introduced by the EDP module of COMSOL software. 


\subsection{Porous models}

In COMSOL, the general form of PDE (for a temporal analysis) must be expressed in the following matrix form

$$
\boldsymbol{\Gamma} \cdot \nabla=\mathbf{F}
$$

where $\boldsymbol{\Gamma}$ is the matrix of the flux vectors and $\mathbf{F}$ is the right part of the vector. In Cartesian coordinates, the gradient/divergence operator vector $\nabla$ is defined as follows

$$
\nabla=\left[\begin{array}{l}
\frac{\partial}{\partial x} \\
\frac{\partial}{\partial y}
\end{array}\right]
$$

\subsubsection{Leclaire's formulation}

If we adapt Leclaire's formulation, Eqs. (3.1), to the EDP form in COMSOL, we obtain the following equations

$$
\boldsymbol{\Gamma}=\left[\begin{array}{ll}
\frac{\partial z}{\partial x} & \frac{\partial z}{\partial y} \\
\frac{\partial w_{s}}{\partial x} & \frac{\partial w_{s}}{\partial y} \\
\frac{\partial w}{\partial x} & \frac{\partial w}{\partial y}
\end{array}\right] \quad \mathbf{F}=\left[\begin{array}{c}
\frac{1}{D+\alpha^{2} M h^{3} / 12}\left(q+h \omega^{2}\left(\rho w_{s}+\rho_{f} w\right)\right) \\
\frac{1}{\alpha M h}\left(\Delta P-h \omega^{2}\left(\rho_{f} w_{s}+m w\right)\right) \\
z
\end{array}\right]
$$

\subsubsection{The mixed formulation}

If we adapt „the mixed formulation”, equations (3.4), to the EDP form of COMSOL, we obtain the following equations

$$
\boldsymbol{\Gamma}=\left[\begin{array}{l}
\Gamma_{i j} \\
\Gamma_{4 i}
\end{array}\right]=\left[\begin{array}{c}
\mu^{S}\left(u_{i / j}+u_{j / i}\right)+\widetilde{\lambda}^{S} u_{k / k} \delta_{i j} \\
p_{/ i}
\end{array}\right] \quad \mathbf{F}=\left[\begin{array}{c}
F_{i} \\
F_{4}
\end{array}\right]=\left[\begin{array}{c}
-\omega^{2} \rho_{e} u_{i}-\gamma p_{/ i} \\
-\omega^{2} \frac{\widetilde{\rho}_{22}}{\widetilde{\lambda^{f}}} p+\omega^{2} \frac{\widetilde{\rho}_{22}}{\phi^{2}} u_{i / i}
\end{array}\right]
$$

\subsection{Geometry}

The geometry of the structure used in the numerical simulation is represented by a coupled porous plate (Fig. 5) with dimensions $a=b$. Thickness of the porous plate is $h p$, of the elastic plate $h s$. The system is loaded by the imposed displacement.

\subsection{Input parameters}

The input parameters are the gear system parameters: motor torque $C_{m}$ and speed $N_{m}$, bearing and shaft stiffnesses $k x_{s}, k y_{s}, k \theta_{s}$, teeth number, width and module $Z, b, m$, average mesh stiffness $k_{c 1}$, contact ratio $\varepsilon_{\alpha 1}$, pressure angle $\alpha$ and 9 poroelastic parameters: porosity $\phi$, tortuosity $\alpha_{\infty}$, flow resistivity $\sigma$, thermal and viscous characteristic dimensions of pores, modulus of elasticity $\Lambda$ and $\Lambda^{\prime}$, density of the skeleton $\rho_{1}$, skeleton Poisson's coefficient $\nu$, damping coefficient $\eta$ and the skeleton elasticity modulus $E$. 


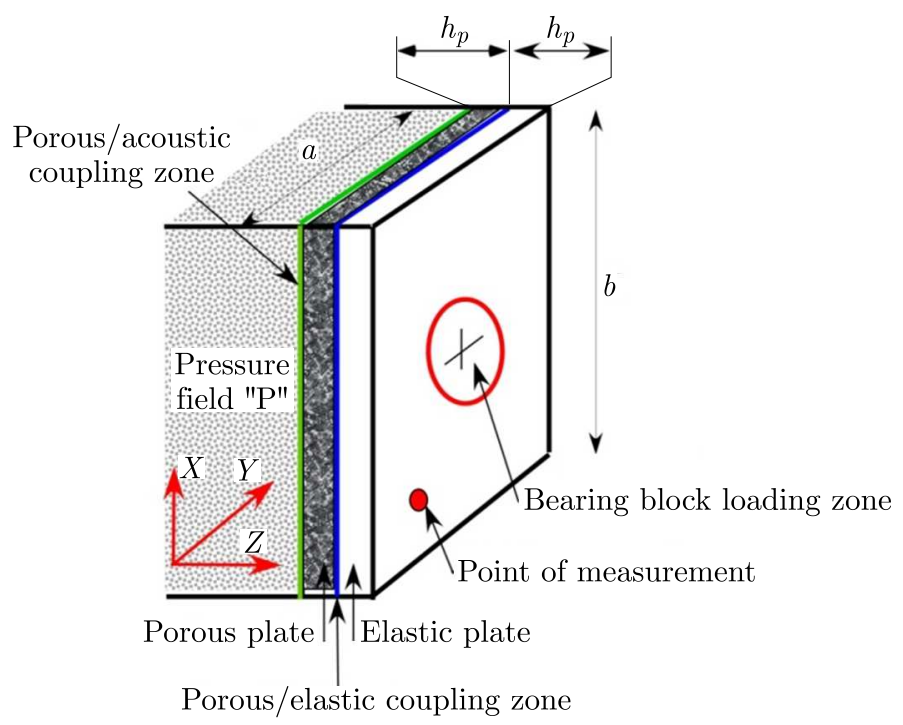

Fig. 5. System of co-ordinates in the plate

\subsection{Output parameters}

The first output is the normal incidence transmission loss $T_{L}$, as introduced by Rossing (2007)

$$
T_{L}=10 \log \frac{1}{\left|T_{a}\right|^{2}}
$$

where $\left|T_{a}\right|^{2}$ is the normal incidence power transmission coefficient for an anechoically-terminated sample, that is the ratio of the sound power transmitted by the sample to the sound power incident on the sample. In the case of perfectly anechoic termination $T_{a}=C / A$

$$
A=\frac{j\left(P_{1} \mathrm{e}^{j k x_{2}}-P_{2} \mathrm{e}^{j k x_{1}}\right)}{2 \sin \left[k\left(x_{1}-x_{2}\right)\right]} \quad C=\frac{j\left(P_{3} \mathrm{e}^{j k x_{4}}-P_{4} \mathrm{e}^{j k x_{3}}\right)}{2 \sin \left[k\left(x_{3}-x_{4}\right)\right]}
$$

with $P_{1}$ to $P_{4}$ are complex sound pressures at $x_{1}$ to $x_{4}$, and $k$ is the wave number.

The second output is the bearing block load

$$
F_{b}=K_{x 3} x_{3}+K_{y 3} y_{3}
$$

$x$ and $y$ are bearing displacements, $K$ is the bearing stiffness and $F_{b}$ is the bearing block load.

\subsection{Boundary conditions}

The boundary conditions for EDP in COMSOL in their general form are as follows

$$
\mathbf{0}=\mathbf{R} \quad-\mathbf{\Gamma n}=\mathbf{G}+\left[\frac{\partial \mathbf{R}}{\partial u}\right]^{\mathrm{T}} \mu
$$

The vector $\mathbf{R}$ and matrix $\boldsymbol{\Gamma}$ may be functions of the spatial co-ordinates with $\mathbf{n}$ being the normal unit vector leaving the boundary surface. These are the boundary conditions of Dirichlet and Neumann, respectively. The term $\mu$ in the Neumann boundary conditions is synonymous with the Lagrange multiplier.

There are several boundary conditions to be respected since there are two clamped coupled plates with four sides and poroelastic/acoustic as well as poroelastic/elastic coupling zones. Using the Biot-Allard formulation, the boundary conditions are discussed below. 
- Imposed pressure field

The imposed pressure field $\bar{p}$ on the boundary of the porous medium allows us to write the following relations

$$
\sigma_{i j}^{t} n_{j}=-\bar{p} n_{i} \quad p=\bar{p}
$$

which express the continuity of the total normal stress and continuity of pressure across the interface of the border. The total stress is equal to

$$
\begin{aligned}
\sigma_{i j}^{t} & =\sigma_{i j}^{S}+\sigma_{i j}^{f}=\sigma_{i j}^{S}-\phi p \delta_{i j}=\widehat{\sigma}_{i j}^{S}-\phi\left(1+\frac{\widetilde{\lambda}^{f S}}{\widetilde{\lambda}^{f}}\right) p \delta_{i j} \\
& =\mu^{S}\left(u_{i / j}+u_{j / i}\right)+\widetilde{\lambda}^{S} u_{k / k} \delta_{i j}-\phi\left(1+\frac{\widetilde{\lambda}^{f S}}{\widetilde{\lambda}^{f}}\right) p \delta_{i j}
\end{aligned}
$$

Using the second boundary condition of Eq. (4.9), the first one can be expressed as follows

$$
-\left[\mu^{S}\left(u_{i / j}+u_{j / i}\right)+\tilde{\lambda}^{S} u_{k / k} \delta_{i j}\right] n_{j}=\left[1-\phi\left(1+\frac{\tilde{\lambda}^{f S}}{\widetilde{\lambda}^{f}}\right)\right] \bar{p} n_{i}
$$

After identification, the terms $\mathbf{R}$ and $\mathbf{G}$ are as follows

$$
\mathbf{R}=\left[\begin{array}{l}
R_{i} \\
R_{4}
\end{array}\right]=\left[\begin{array}{c}
0 \\
\bar{p}-p
\end{array}\right] \quad \mathbf{G}=\left[\begin{array}{l}
G_{i} \\
G_{4}
\end{array}\right]=\left[\begin{array}{c}
\left.1-\phi\left(1+\frac{\tilde{\lambda}^{f S}}{\widetilde{\lambda}^{f}}\right)\right] \bar{p} n_{i} \\
0
\end{array}\right]
$$

When a portion of the surface of the porous medium is coupled to an infinite acoustic medium, the condition of a free edge can be applied. This is assuming that $\bar{p}=0$.

- Imposed displacement field

In the case of the imposed displacement field $\bar{u}_{i}$, the boundary conditions can be expressed by

$$
u_{i}=\bar{u}_{i} \quad v_{i} n_{i}-u_{i} n_{i}=0
$$

The first term in Eq. (4.13) expresses the continuity between the imposed displacements and the solid phase displacements, while the second term describes the continuity of the normal displacement between the fluid and solid phase. In this second condition, it is necessary to replace the displacement of the fluid phase by the fluid pressure

$$
v_{i}=\frac{\phi}{\omega^{2} \widetilde{\rho}_{22}} p_{/ i}-\frac{\widetilde{\rho}_{12}}{\widetilde{\rho}_{22}} u_{i}
$$

which yields

$$
p_{i i} n_{i}=\frac{\omega^{2}}{\phi}\left(\widetilde{\rho}_{12}+\widetilde{\rho}_{22}\right) u_{i} n_{i}
$$

such as

$$
\frac{\omega^{2}}{\phi}\left(\widetilde{\rho}_{12}+\widetilde{\rho}_{22}\right)=\frac{\omega^{2}}{\phi}\left(\rho_{12}+\rho_{22}\right)=\omega^{2} \rho_{0}
$$

After identification, the terms $\mathbf{R}$ and $\mathbf{G}$ are as follows

$$
\begin{array}{lc}
R_{i}=\bar{u}_{i}-u_{i} & R_{4}=0 \\
G_{i} & =0 \quad G_{4}=-\frac{\omega^{2}}{\phi}\left(\widetilde{\rho}_{12}+\widetilde{\rho}_{22}\right) u_{i} n_{i}
\end{array}
$$

Applying that $\bar{u}_{i}=0$ implies the fact that our porous domain is embedded to a rigid wall. 
- Acoustic - poroelastic coupling

In this case, the equations for continuity of the total normal stresses, acoustic pressure and fluid flow are as follows

$$
\begin{aligned}
& \sigma_{i j}^{t} n_{j}=-p^{a} n_{j} \quad p=p^{a} \\
& (1-\phi) u_{i} n_{i}+\phi v_{i} n_{i}=\frac{1}{\rho_{0} \omega^{2}} \nabla p^{a} n_{i}
\end{aligned}
$$

where $p^{a}$ is pressure in the acoustic medium, $\rho_{0}$ its density and $\sigma^{t}$ the total stress tensor in the poroelastic material. The vectors $\mathbf{G}$ and $\mathbf{R}$ will have the following components

$$
\begin{aligned}
R_{i} & =0 \quad R_{4}=p-p^{a} \\
G_{i} & =\left[1-\phi\left(1+\frac{\widetilde{\lambda}^{f S}}{\widetilde{\lambda}^{f}}\right)\right] p^{a} n_{i} \quad G_{4}=0
\end{aligned}
$$

In addition, the continuity of the fluid flow at the coupling interface can be expressed as an imposed acceleration on the fluid in the acoustic environment. Replacing $v_{i}$ by its expression, the normal acceleration can be obtained by

$$
\frac{1}{\rho_{0}} \nabla p^{a} n_{i}=\omega^{2}\left[u_{i} n_{i}\left(1-\phi\left(1+\frac{\widetilde{\rho}_{12}}{\widetilde{\rho}_{22}}\right)\right)\right]+\omega^{2}\left[\nabla p n_{i}\left(\frac{\phi^{2}}{\omega^{2} \widetilde{\rho}_{22}}\right)\right]
$$

For the Leclaire formulaion, a boundary condition can be considered. It is discussed below

- Clamped plate

At the boundary conditions, an embedding condition is introcuced

$$
w_{s}=0 \quad U_{f}=0
$$

The relative solid-fluid displacement is defined as follows

$$
w=\phi\left(U_{f}-w_{s}\right) \quad U_{f}=\frac{1}{\phi} w+w_{s}
$$

where $w_{s}$ is the solid displacement and $U_{f}$ is the fluid displacement.

Subsequently, $\mathbf{R}$ and $\mathbf{G}$ are expressed by

$$
\mathbf{R}=\left[\begin{array}{c}
w_{s} \\
\frac{1}{\phi} w+w_{s} \\
0
\end{array}\right] \quad \mathbf{G}=\left[\begin{array}{l}
0 \\
0 \\
0
\end{array}\right]
$$

The loading conditions $q$ and $\Delta P$ are fixed according to the type of solicitation (pressure, force,...). For the surface pressure, a value of 0.1 is assumed

$$
\Delta P=q=0.1 \text { bars }
$$

\section{Study case}

The numerical parameters of the two-stage gear system are summarized in Table 1.

Table 3 describes numerical values of parameters of the poroelastic materials. 
Table 1. Geared transmission parameters

\begin{tabular}{|c|c|c|}
\hline External inputs & Motor torque and speed & $C_{m}=1000 \mathrm{Nm}, N_{m}=3000 \mathrm{tr} / \mathrm{mn}$ \\
\hline $\begin{array}{c}\text { Structure } \\
\text { characteristics }\end{array}$ & Bearing and shaft stiffness & $x_{s}=k y_{s}=10^{9} \mathrm{~N} / \mathrm{m}$, \\
$k \theta_{s}=10^{5} \mathrm{Nm} / \mathrm{rad}$
\end{tabular}

\begin{tabular}{|l|c|c|}
\hline \multicolumn{3}{|c|}{ Gear characteristics material: $42 \mathrm{CrMo} 4, \rho=7860 \mathrm{~kg} / \mathrm{m}^{3}$} \\
\hline \hline & First stage & Second stage \\
\hline Teeth width and module $[\mathrm{mm}]$ & $b=20, m=4$ & $b=20, m=4$ \\
\hline Teeth number & $Z(12)=26, Z(21)=39$ & $Z(12)=26, Z(21)=39$ \\
\hline Average mesh stiffness & $k_{c 1}=1.4 \cdot 10^{8} \mathrm{~N} / \mathrm{m}$ & $k_{c 2}=1.4 \cdot 10^{8} \mathrm{~N} / \mathrm{m}$ \\
\hline Contact ratio and pressure angle & $\varepsilon_{\alpha 1}=1.57, \alpha=20^{\circ}$ & $\varepsilon_{\alpha 2}=1.53, \alpha=20^{\circ}$ \\
\hline
\end{tabular}

Table 2. First eigenfrequency of the geared transmission

\begin{tabular}{|c|c|c|c|c|c|c|}
\hline$\omega_{i}[\mathrm{rad}]$ & 1823 & 4095 & 6016 & 16063 & 17353 & 27365 \\
\hline$f_{i}[\mathrm{~Hz}]$ & 290 & 652 & 957 & 2557 & 2763 & 4357 \\
\hline
\end{tabular}

Table 3. Poroelastic parameters for validation of the models

\begin{tabular}{|c|c|c|}
\hline Parameter & Unity & Porous material \\
\hline \hline$\rho_{1}$ & $\mathrm{~kg} / \mathrm{m}^{3}$ & 90 \\
\hline$\phi$ & - & 0.7 \\
\hline$\sigma$ & $\mathrm{Ns} / \mathrm{m}^{4}$ & 22250 \\
\hline$\alpha_{\infty}$ & - & 1.3 \\
\hline$\nu$ & - & 0.05 \\
\hline$\Lambda$ & $\mu \mathrm{m}$ & 75 \\
\hline$\Lambda^{\prime}$ & $\mu \mathrm{m}$ & 87 \\
\hline$E$ & $\mathrm{~N} / \mathrm{m}^{2}$ & 2980000 \\
\hline$\eta$ & - & 0.12 \\
\hline
\end{tabular}

\subsection{Porous plate effect on vibration level}

Figure 6 shows the displacement along the axis $x$ of the output bearing at the housing cover. The displacement amplitude is about $2 \cdot 10^{-6}$. The periodicity of the bearing displacement comes from domination of the gearmesh frequency.

Figure 7 shows that the RMS bearing displacement increases with the meshing frequency as it is shown in Fig. 8. The results show that the gearmesh frequency and its harmonics dominate the RMS bearing displacement with higher amplitudes when the gearmesh frequency or one of its harmonics is close to the eigenfrequency. The first peak is close to the first eigenfrequency $(290 \mathrm{~Hz})$ the second one is close to the third eigenfrequency $(957 \mathrm{~Hz})$. The third peak is close to the sum of the first and the third eigenfrequency $(1608 \mathrm{~Hz})$.

Figure 9 shows that the gearmesh frequency and its harmonics dominate the point plate displacement. The absence of a negative displacement is due to the elastic effect of the plate at the measurement point. Due to the same reason, there are no positive displacements in the other half of the plate.

As it is shown in Fig. 10, the gearmesh frequency dominates the point plate displacement. The absence of a negative displacement is due to the elastic effect of the plate at the measurement point. Due to the same cause, there are no positive displacements in the other half of the plate. 


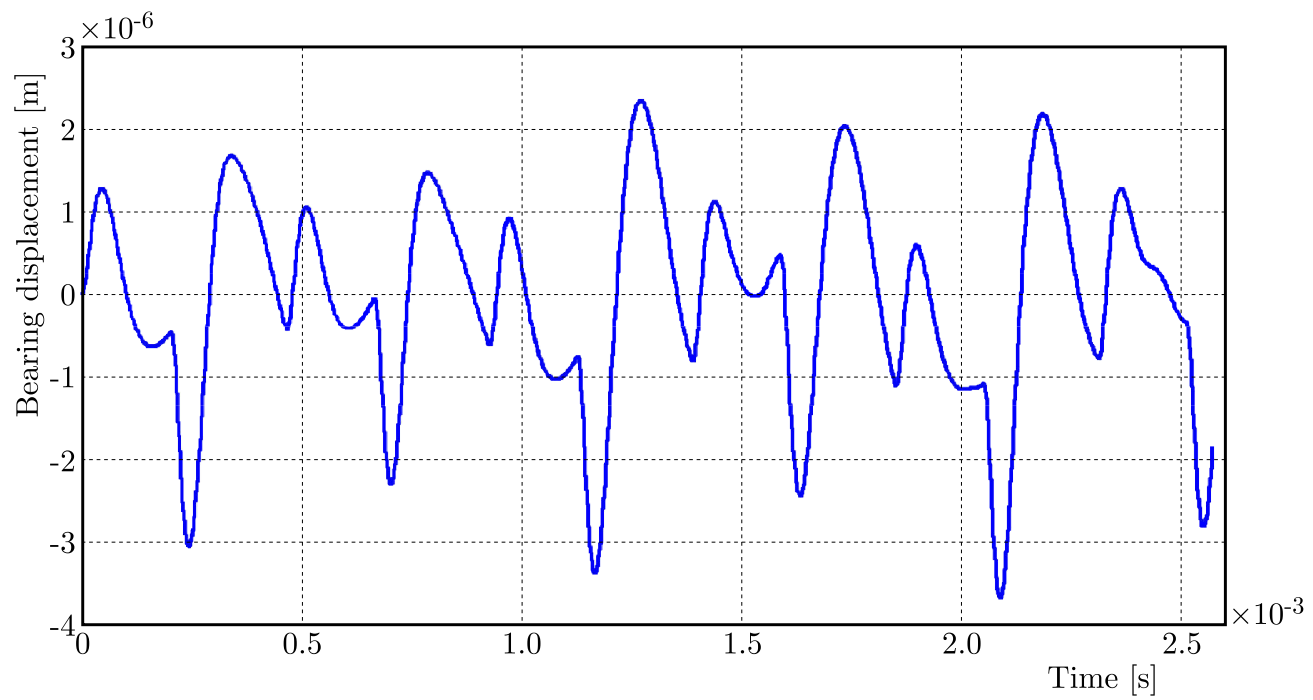

Fig. 6. Output bearing displacement in the $x$ direction

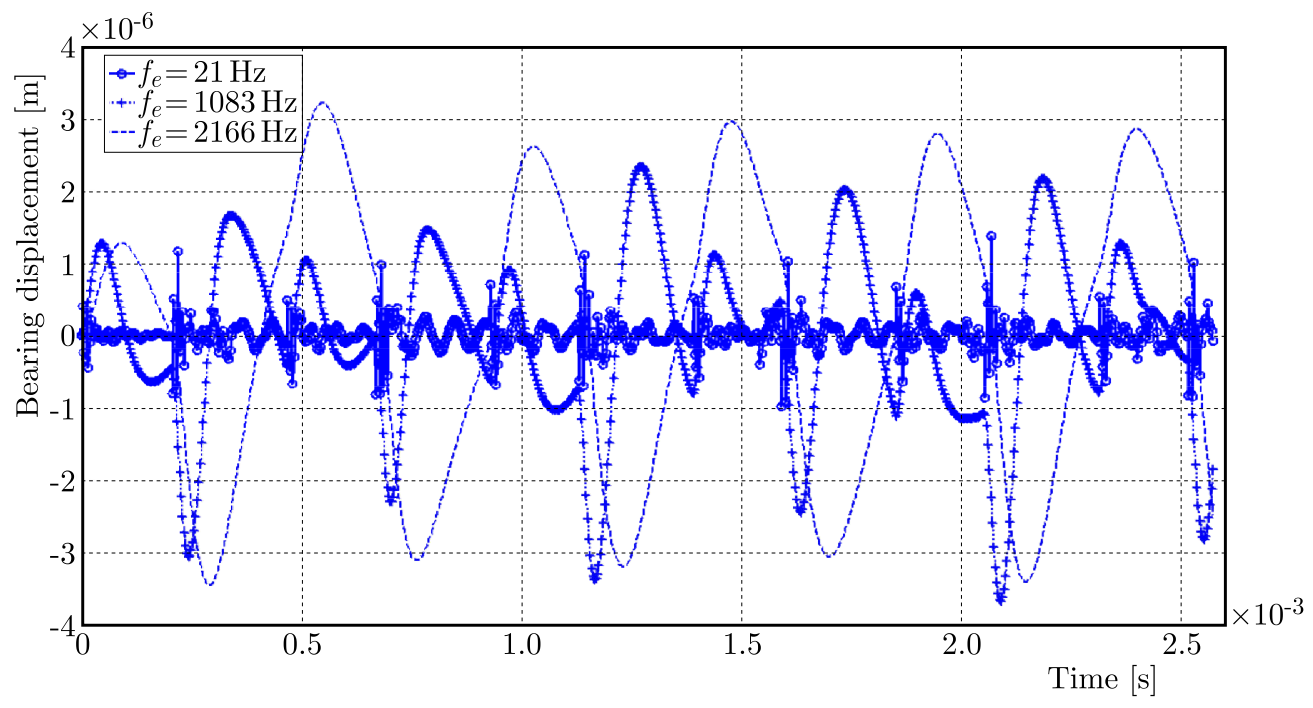

Fig. 7. Output bearing displacement in the $x$ direction for three gearmesh frequencies

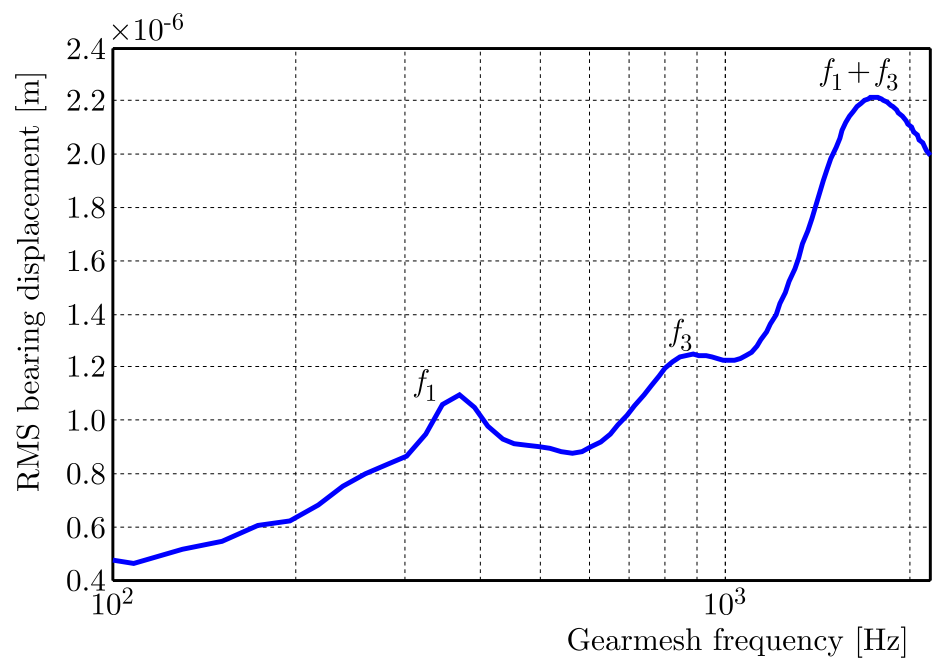

Fig. 8. RMS bearing displacement 

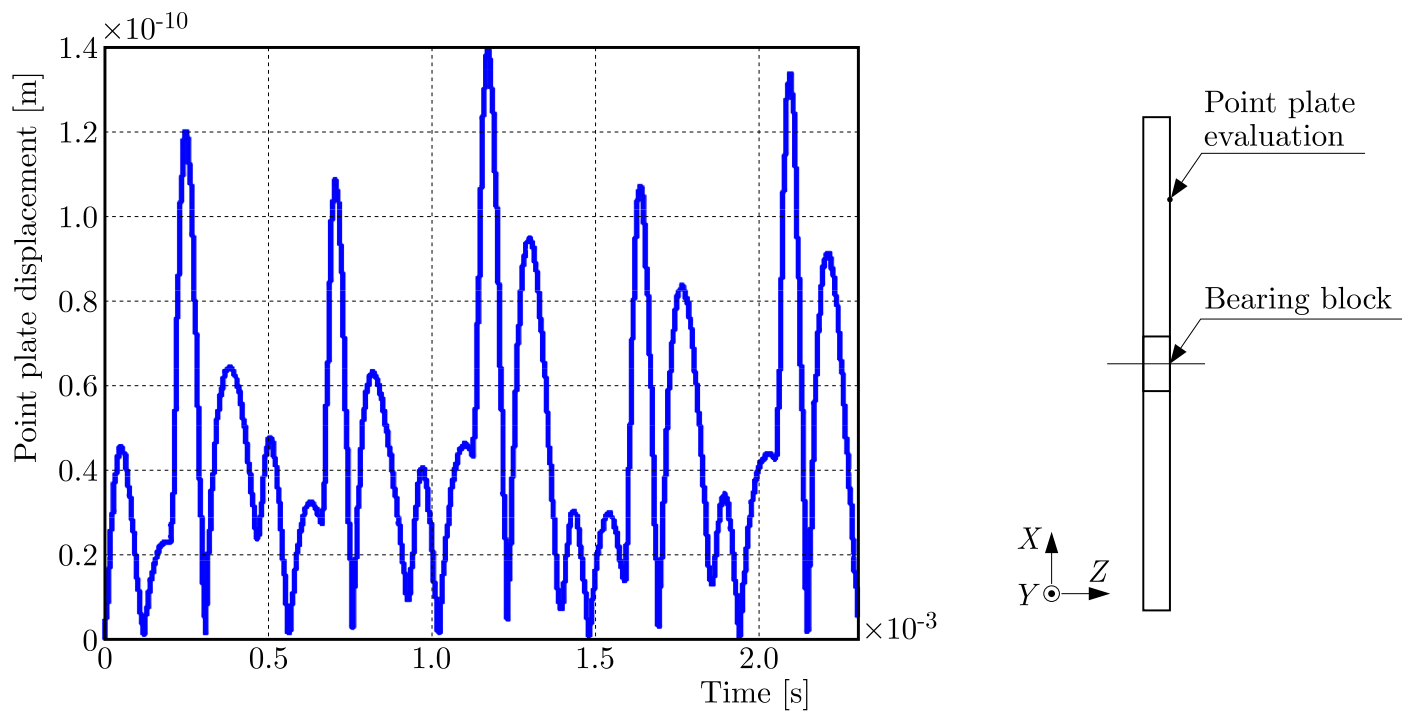

Fig. 9. Displacement along the axis $x$ at a point with coordinates $(0.15,0.24)$ on the elastic plate
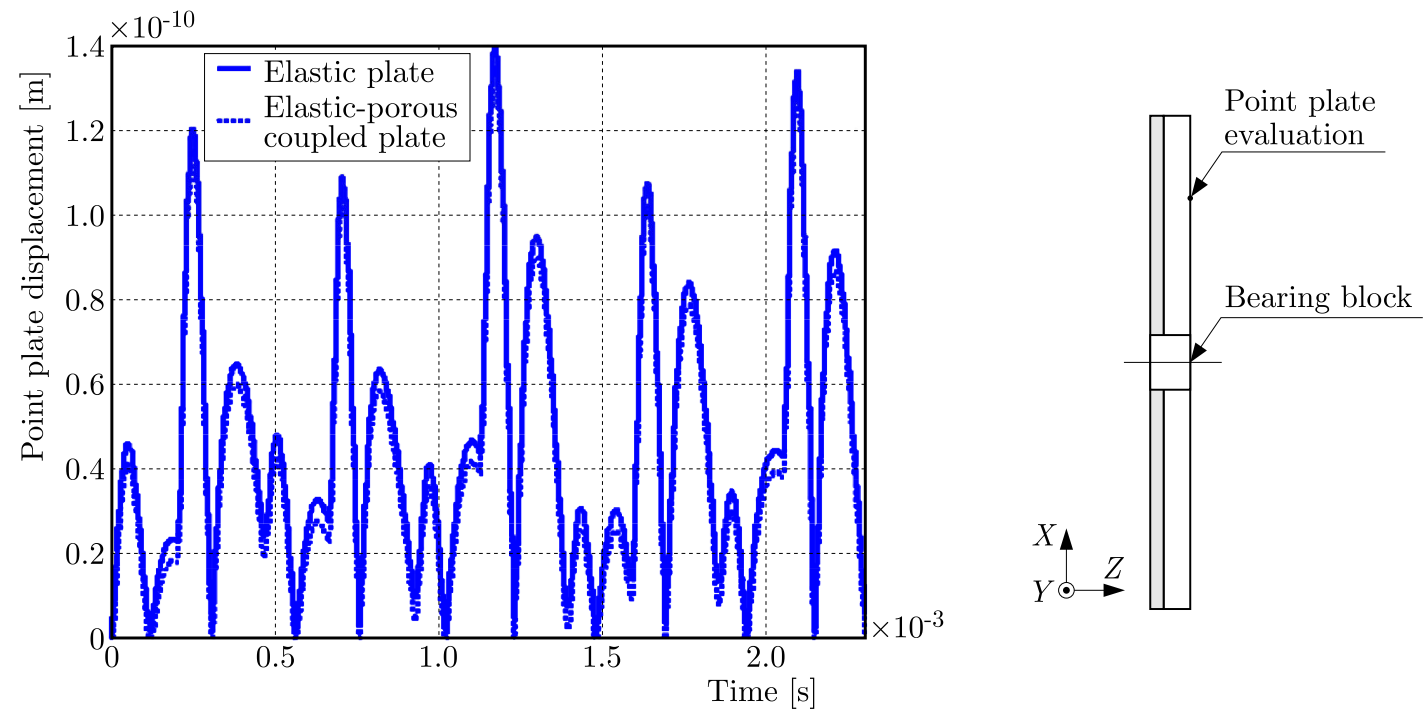

Fig. 10. Point displacements (solid line: elastic plate, dashed: elastic and porous coupled plate)

\subsection{Acoustic effect of the porous plate}

Figure 11 shows the transmission loss $T_{L}$ of the elastic-porous coupled plate. The calculation is conducted for the porous plate with a characteristic defined in Table 3 and thickness $10 \mathrm{~mm}$. $T_{L}$ increases along the frequency axis and is dominated by the resonance frequency of the plate where $T_{L}$ decreases with the frequency converging to $67 \mathrm{~dB}, 54 \mathrm{~dB}$ and $83 \mathrm{~dB}$ at, respectively, natural frequencies $620 \mathrm{~Hz}, 1240 \mathrm{~Hz}$ and $1900 \mathrm{~Hz}$. Figure 11 shows the dependence of the sound transmission loss on the flow resistivity which is one of the characteristic of the porous material but is still dominated by the natural frequencies.

\section{Conclusion}

A resolution method to determine the porous plate effect on a gearbox hosing cover is discusseg in the paper. The developed model depends on several parameters: gearbox and porous plates parameters. It is found that coupling of the porous plate to the housing cover reduces the 


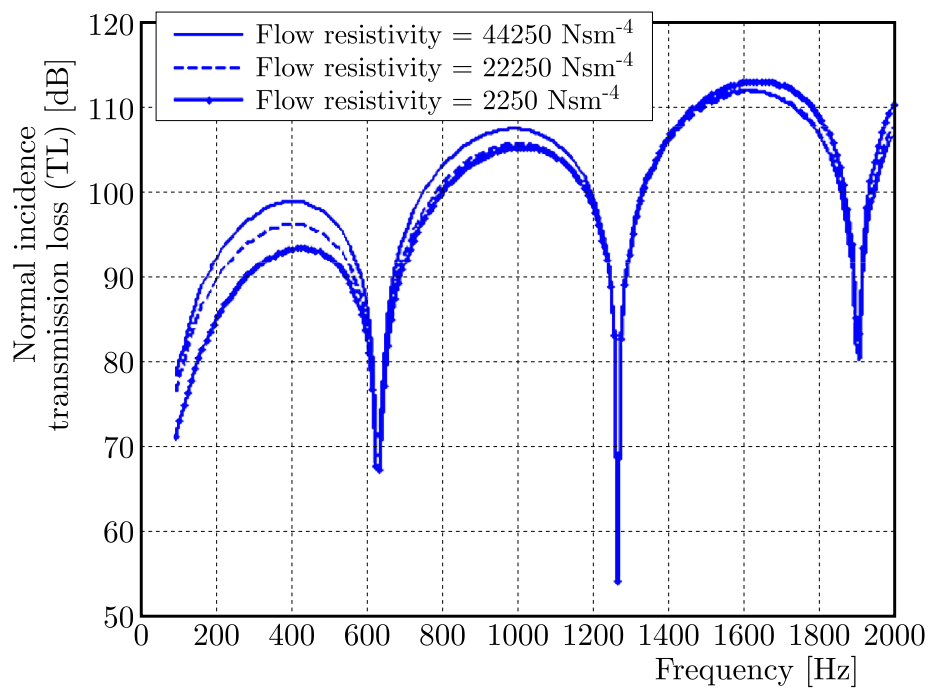

Fig. 11. Sound transmission loss $T_{L}$ for different flow resistivity $\sigma$

vibration level and is dominated by the gearmesh frequency. For the acoustic effect, poroelastic materials have major capacity to mitigate the noise level caused by the geared transmission. The vibration and the acoustic behavior are heavily dependent on poroelastic characteristics. These results were validated by Tewes (2005), who computed the transmission loss of an infinite double wall partition for various angles of incidence and for various mass ratios. The developed method helps one to make decisions in the robust design and lessens the enormous computing time.

\section{References}

1. Allard J.F., 1993, Propagation of Sound in Porous Media, Elsevier Science, Essex, UK

2. Allard J.F., Champoux Y., 1992, New empirical equations for sound propagation in rigid frame fibrous materials, Journal of the Acoustical Society of America, 91, 3346-3353

3. Allard J.F., Herztog P., Lafarge D., 1993, Recent topics concerning the acoustics of fibrous and porous materials, Applied Acoustics, 39, 3-21

4. Atalla N., Panneton R., Debergue P., 1998, A mixed displacement-pressure formulation for poroelastic materials, Journal of the Acoustical Society of America, 104, 3, 1444-1452

5. Aziz S.M.A., SeIrg A., 1994, Parametric study of frictional noise in gear, Wear, 176, 1, 25-28

6. Biот M.A., 1962, Mechanics of deformation and acoustics propagation in porous media, Journal of Applied Physics, 33, 1482-1498

7. Champoux Y., Allard J.F., 1991, Dynamic tortuosity and bulk modulus in air-saturated porous media, Applied Physics, 70, 4, 1975-1979

8. Champoux M., Stinson M.R., 1992, On acoustical models for sound propagation in rigid frame porous materials and the influence of shape factors, Journal of the Acoustical Society of America, 92, 1120-1131

9. Chedly S., Chettah A., Bonnnefoy H., Ichchou M., 2008, A robust shock and noise models for the manufacturing of moulded LDPE foams, Polymer Engineering and Science

10. Delany M.E., Bazley E.N., 1970, Acoustic properties of fibrous absorbent materials, Applied Acoustics, 3, 105-116

11. Guezzen S., 2004, Vibro-acoustic behavior study of rib structures (in French), Thesis, INSA Lyon, France, http://theses.insa-lyon.fr/publication/2004ISAL0092/these.pdf 
12. Hentati T., Bouazizi L., Taktak M., Trabelsi H., Haddar M., 2016, Multi-levels inverse identification of physical parameters of porous materials, Applied Acoustics, 108, 26-30

13. Horoshenkov K.V., Swift M.J., 2001, The acoustic properties of granular materials with pore size distribution close to log-normal, Journal of the Acoustical Society of America, 110, 2371-2378

14. Houjoh H., Umezawa K., 1992, A generation mechanism of aerodynamic sound of spur gears, Proceedings of the 6th International Power Transmission and Gearing Conference, Scottsdale, 2, 597-604

15. Houser D., 1991, Gear Noise, Dudley's Gear Handbook, 2nd Edition, McGraw Hill

16. Johnson D.L., Koplik J., Dashen R., 1987, Theory of dynamic permeability and tortuosity in fluid-saturated porous media, Journal of Fluid Mechanics, 176, 379-402

17. Lafarge D., Lemarinier P., Allard J.F., Tarnow V., 1997, Dynamic compressibility of air in porous structures at audible frequencies, Journal of the Acoustical Society of America, 102, 4, $1995-2006$

18. Leclaire P., Horoshenkov V., Cummings A., 2001, Transverse vibrations of a thin rectangular porous plate saturated by a fluid, Journal of Sound and Vibration, 247, 1-18

19. Miller A.J., 1999, A new wavelet basis for the decomposition of gear motion error signals and its application to gearbox diagnostics, M.Sc. Thesis, The Pennsylvania State University, Pennsylvania, PA, USA

20. Othmani C., Hentati T., Taktak M., Elnady T., Haddar M., 2015, Effect of liner characteristics on the acoustic performance of duct systems, Technical Note, 40, 1, 117-127

21. Phong V., 2012, Acoustic transmission loss of perforated plates, AIAA/CEAS Aeroacoustics Conference, Colorado Springs

22. Phong V., 2015, Normal incidence acoustic transmission loss of perforated plates with Bias flow, 53rd AIAA Aerospace Sciences Meeting, Kissimmee, Florida

23. Putra A., Ismail A.Y., Ramlan R., Ayob Md.R., Py M.S., 2013, Normal incidence of sound transmission loss of a double-leaf partition inserted with a microperforated panel, Advances in Acoustics and Vibration, ID 216493

24. Rossing T., 2007, Handbook of Acoustic, Springer, LLC New York, ISBN 978-0-387-30425-0

25. Sibe A., 1997, Study of the acoustic transparency of transmission housings of gears (in French), Thesis, http://bibli.ec-lyon.fr/exl-doc/TH_T1701_pducret.pdf

26. Tewes S., 2005, Active trim panel attachments for control of sound transmission through aircraft structures, Dissertation, https://mediatum.ub.tum.de/doc/601937/document.pdf

27. Umnova O., Attenborough K., Li K.M., 2001, A cell model for the acoustical properties of packings of spheres, Acta Acustica, 87, 226-235

28. Velex P., 1988, Contribution to the analysis of the dynamic behavior of parallel axis gears (in French), Thesis, INSA Lyon, France, No. 88, ISAL 0032, 188 p.

29. Velex P., Sainsot P., 2002, An analytical study of tooth friction excitations in errorless spur and helical gears, Mechanism and Machine Theory, 37, 641-658

30. Walha L., Fakhfakh T., Haddar M., 2009, Nonlinear dynamics of a two-stage gears system with mesh stiffness fluctuation, bearing flexibility and backlash, Mechanism and Machine Theory, 44, 1058-1069

31. Wilson D.K., 1997, Simple relaxational models for the acoustical properties of porous media, Applied Acoustics, 50, 171-188

32. Zielinski T.G., Gallad M.A., IChChou M., 2012, Fully coupled finite-element modeling of active sandwich panels with poroelastic core, Journal of Vibration and Acoustics, 134, 021007, $1-10$ 